\title{
A five year retrospective study on maternal and perinatal outcome in pregnancy after cardiac surgery
}

\author{
Swati Rathore', Ravi Shankar ${ }^{2 *}$, Annie P. Vijjeswarapu ${ }^{1}$, Anuja Abraham ${ }^{1}$, Bijesh Yadav ${ }^{3}$
}

${ }^{1}$ Department of Obstetrics and Gynecology, ${ }^{2}$ Department of Cardiothoracic Surgery, ${ }^{3}$ Department of Biostatistics, Christian Medical College, Vellore, Tamil Nadu, India

Received: 01 March 2018

Accepted: 28 March 2018

\section{*Correspondence:}

Dr. Ravi Shankar,

E-mail: ravixshanker@gmail.com,

Copyright: $\odot$ the author(s), publisher and licensee Medip Academy. This is an open-access article distributed under the terms of the Creative Commons Attribution Non-Commercial License, which permits unrestricted non-commercial use, distribution, and reproduction in any medium, provided the original work is properly cited.

\begin{abstract}
Background: Pregnancy is a hypercoaguable state with physiological haemodynamic changes occurring during pregnancy. There is a progressive increase in intravascular volume in second trimester of pregnancy and increase in cardiac output. Pregnancy makes a significant impact on cardiovascular system. It is important to evaluate and study the effect of pregnancy on women with surgically corrected heart conditions so as to preempt potential complications. Methods: This is a retrospective study of patients with prior history of cardiac surgery and their pregnancy outcomes in a tertiary center of Southern India over a period of five years from January 2011 to December 2016.

Results: In this study, descriptive statistical analysis was done in 87 women with pregnancy following cardiac surgery. $58.6 \%$ were nulliparous. Around $52 \%$ had associated obstetric risk factors. The most common cardiac surgery in this population was Mitral valve replacement (40.2\%) and Atrial septal defect closure (37.9\%). Women belonged to NYHA class I in $90.8 \%$ of cases. $58.6 \%$ had vaginal delivery and 36.8\% had caesarean section. 6 women had postpartum haemorrhage which was medically managed, and 6 women needed ICU care.74.7\% women had term deliveries. $18.4 \%$ of the babies were less than $2.5 \mathrm{~kg}$ weight at birth. 13 babies required Neonatal ICU care.

Conclusions: Maternal and neonatal outcome mainly depends on the functional cardiac status of women before conception. In this study we emphasize on the importance of multidisciplinary team approach involving cardiologist, obstetrician and neonatologist in the management of women with prior cardiac surgery.
\end{abstract}

Keywords: Cardiac surgery, Functional cardiac status, Pregnancy outcome

\section{INTRODUCTION}

Pregnancy is a hypercoaguablestate with physiological haemodynamic changes occurring during pregnancy. ${ }^{1,2}$ There is a progressive increase in intravascular volume in second trimester of pregnancy and increase in cardiac output. $^{3}$

Pregnancy makes a significant impact on cardiovascular system. It is important to evaluate and study the effect of pregnancy on women with surgically corrected heart conditions so as to preempt potential complications. Pregnancies complicated with maternal cardiac diseases have an incidence of $1-4 \%$. Most of these can be managed with lifestyle changes and medical management. Pregnancy following cardiac surgery puts both mother and fetus at risk. Maternal mortality rate can be as high as 10-30\%. ${ }^{4}$ Pregnancy among women with mechanical prosthetic valve is associated with arisk of maternal death of between 1 and $4 \% .^{5}$ Thus patients with artificial heart valves need an enhanced level of care during pregnancy and labour. ${ }^{6}$ Mortality is mainly due to thrombotic complications. Neither surgical nor medical treatment is ideal for pregnant women with acute prosthetic heart valve dysfunction. Hence appropriate time and the mode of delivery prior to the therapy when 
the gestation age is beyond 32 weeks is preferred. Management also depends on maternal status. There is no contraindication for pregnancy in patients with NYHA (New York heart association) class I and II after cardiac surgery. However physiological changes in pregnancy put strain on cardiovascular system and many times it is hard to predict the consequences. In NYHA class III and IV, serious adverse cardiac events can be as high as $57 \% .^{7}$ Patients with cardiac disease because of impaired circulation state may have hypoxia of myometrium and hence the risk of premature labour is high. ${ }^{8}$ Pulmonary edema, cardiac arrthymias, postpartum haemorrhage and thromboembolic events increase the maternal morbidity. ${ }^{8}$ The maternal and fetal outcome in women who had prior cardiac surgery can be improved with appropriate medical care. Awareness to general population, knowledge about progress of disease and effect of pregnancy on corrected heart conditions per se should be discussed in detail with the couple and family members prior to conception.

\section{METHODS}

This isa retrospective study of patients with prior history of cardiac surgery and their pregnancy outcomes in a tertiary center of Southern India over a period of five years from Jan 2011 to Dec 2016. Eighty seven pregnant women were studied and data was analyzed retrospectively for maternal and neonatal outcome. Variables reviewed were demographic data like maternal age, parity, successful live birth, obstetrics risk factors such as gestational hypertension, gestational diabetes on treatment, anemia and previous caesarean section, type of cardiac surgery, mode of delivery, postpartum complications. Neonatal outcomes noted were as follows : birth weight, APGAR score, need for Neonatal ICU admission. Types of cardiac surgery in this study population were mitral valve replacement, aortic valve repair, mitral and aortic valve repair, atrial septal defect and ventricular septal defect repair, repair of Tetralogy of Fallot. NYHA functional class [I to IV] of the women was documented in the study. Progression of functional class of NYHA from class 1 to 3 and 4 makes potential difference in both maternal and fetal outcome. Descriptive statistical analysis was done.

\section{RESULTS}

Descriptive statistical analysis of 87 pregnant women with previous history of cardiac surgery was done and used to describe the data. Maternal age was ranging from 20 years to $39 y e a r s$. The mean age recorded in the study group was 25.6 years. Distribution in accordance with parity were as follows $58.6 \%$ were nullipara, $19.5 \%$ were primipara, multiparous were $21.8 \%$. According to the past obstetric score, we found one live child in $31 \%$ and 4.6\% had 2 live children. Among 87 pregnant women, $11.5 \%$ had gestational diabetes and were on medical nutritional therapy or on medication during pregnancy, $6.9 \%$ women had associated gestational hypertension and mild preeclampsia. 10 pregnant women had previous bad obstetric history and 8 women had pregnancy complicated with anemia. $11.5 \%$ women had previous one or two lower segment caesarean sections.47.8\% women had no associated obstetrical risk factors.

Table 1: Type of cardiac surgery done before pregnancy.

\begin{tabular}{|lllll|}
\hline & Frequency & $\%$ & $\begin{array}{l}\text { Valid } \\
\%\end{array}$ & $\begin{array}{l}\text { Cumulative } \\
\%\end{array}$ \\
\hline $\begin{array}{l}\text { ASD } \\
\text { repair }\end{array}$ & 33 & 37.9 & 37.9 & 37.9 \\
\hline $\begin{array}{l}\text { VSD } \\
\text { repair }\end{array}$ & 10 & 11.5 & 11.5 & 49.4 \\
\hline $\begin{array}{l}\text { ASD with } \\
\text { MVR }\end{array}$ & 02 & 2.3 & 2.3 & 51.7 \\
\hline MVR only & 35 & 40.2 & 40.2 & 92.0 \\
\hline $\begin{array}{l}\text { AVR with } \\
\text { MVR }\end{array}$ & 02 & 2.3 & 2.3 & 94.3 \\
\hline TOF repair & 02 & 2.3 & 2.3 & 96.6 \\
\hline AVR & 03 & 3.4 & 3.4 & 100.0 \\
\hline Total & 87 & 100.0 & 100.0 & \\
\hline
\end{tabular}

Most of the women belonged to NYHA class I, $90.8 \%$ and $9.2 \%$ belonged to NYHA class II. In accordance with heart surgery done in the study group $40.2 \%$ had MVR (mitral valve replacement) and the second most common was ASD (atrial septal defect) repair constituting $37.9 \%$.VSD (ventricular septal defect) repair was reported in $11.5 \%$. Pregnant women with prior history of AVR (aortic valve replacement) were 3.4\%. 2.3\% had a combination of AVR and MVR, $2.3 \%$ had ASD with MVR and $2.3 \%$ had TOF (Teratology of Fallot) correction surgery (Table 1).

Table 2: Mode of delivery.

\begin{tabular}{|llll|l|}
\hline & Frequency & $\%$ & $\begin{array}{l}\text { Valid } \\
\%\end{array}$ & $\begin{array}{l}\text { Cumulative } \\
\%\end{array}$ \\
\hline $\begin{array}{l}\text { Normal } \\
\text { delivery }\end{array}$ & 32 & 36.8 & 36.8 & 36.8 \\
\hline Instrumental & 19 & 21.8 & 21.8 & 58.6 \\
\hline LSCS & 32 & 36.8 & 36.8 & 95.4 \\
\hline Abortions & 04 & 4.6 & 4.6 & 100.0 \\
\hline Total & 87 & 100.0 & 100.0 & \\
\hline
\end{tabular}

In patient on Warfarin, as per our hospital protocol heparin was given till 14 weeks of gestation and then was changed over to oral anticoagulant till 37 weeks and then heparin was given till delivery. Heparin was restarted after 12 hours of caesarean section and 6 hours after vaginal delivery. $6.8 \%$ pregnant women with heart surgery were on digoxin, diuretic and penicillin prophylaxis. $31 \%$ were on heparin/oral Anticoagulant medication, $12.6 \%$ women were on both anti-failure and anticoagulant medication.

Other variables studied were the mode of delivery, indication for caesarean section or reasons for operative vaginal deliveries, type of anaesthesia offered, total blood 
loss, puerperial complication. There were 2 spontaneous abortions at 12 and 14 weeks of gestation and stillbirth at 24 and 27 weeks of gestation. $58.6 \%$ women had vaginal delivery, out of this $21.8 \%$ had instrumental delivery involving both forceps and suction cup delivery. Women requiring caesarean deliveries for various indications were $36.8 \%$. Operative procedure was both emergency and elective. Caesarean done electively (13.7\%) for previously scarred uterus in 6 cases and for malpresentation in 5 cases (Table 2). Emergency cesarean was done for non-reassuring fetal status in $8 \%$,second commonest reason for operative delivery was for failed induction in $8 \%, 2.3 \%$ had lower uterine caesarean section for arrest of dilatation, $1.1 \%$ had caesarean done for intrauterine growth restriction with abnormal dopplers and for deteriorating maternal condition in $4.6 \%$ [pulmonary edema, severe preeclampsia with uncontrolled blood pressure leading to maternal compromise resulting in operative deliveries and intensive care unit admission]. General anaesthesia was offered to $14.1 \%$ and spinal anaesthesia to $24 \%$. Blood loss more than a liter was found in 6 women, 4 women had uterine atonicity, traumatic postpartum heamorrhage was found in 2 cases. Postpartum puerperial complication like endomyometritis, urinary tract infection was seen in $3.3 \%$. One woman had supraventricular tachycardia after delivery.

Table 3: Baby weight at delivery.

\begin{tabular}{|lllll|} 
& Frequency & $\%$ & $\begin{array}{l}\text { Valid } \\
\%\end{array}$ & $\begin{array}{l}\text { Cumulative } \\
\%\end{array}$ \\
\hline$<2499$ gm & 16 & 18.4 & 18.4 & 18.4 \\
\hline$>2500$ & 67 & 77.0 & 77.0 & 95.4 \\
\hline NA & 04 & 4.6 & 4.6 & 100.0 \\
\hline Total & 87 & 100.0 & 100.0 & \\
\hline
\end{tabular}

Neonatal parameters studied were birth weight, APGAR score, Neonatal ICU admission. Birth weight after delivery was found to be less than $2.5 \mathrm{~kg}$ in $18.4 \%$ while $77 \%$ of babies' birth weight was more than $2.5 \mathrm{~kg}$ (Table 3). 14 babies were admitted in NICU. Reasons for NICU admission were low APGAR score, respiratory distress syndrome and sepsis.

Authors analyzed maternal variables before and after delivery. The occurrence of spontaneous abortion (upto 20 weeks of gestation), still birth, premature birth (birth upto 36 weeks and 6 days of gestation].75\% women delivered at term [more than 37 weeks of gestation), preterm delivery in $18.3 \%$. Out of this, late preterm between 34 to 36 weeks and 6 days of gestation were $14.9 \%$ and $3.4 \%$ in less than 33 weeks and 6 days

Similarly, maternal ICU admission and subsequent successful pregnancy outcome were studied. ICU care was required for 6 mothers after delivery. Among 87 women with prior history of cardiac Surgery, $37.9 \%$ women had subsequent second successful pregnancy.

\section{DISCUSSION}

Heart disease in pregnancy is an indirectcause of maternal mortality. Rheumatic heart disease, congenital heart disease are more common than ischaemic heart disease and cardiomyopathy. Though there is significant decrease in occurence of rheumatic heart disease, the incidence of cardiac disease in pregnancy remained stable over many years. This is because of the significant increase in pregnancy in women with congenital heart disease. ${ }^{4}$ Advances in cardiac surgery have significantly contributed to better level of functioning of the cardiovascular system and better quality of life for patients with both acquired and congenital heart disease.

Management of pregnant woman following cardiac surgery possess a significant challenge to an obstetrician. Maternal mortality is mainly due to complications like thromboembolic, haemorrhage and heart failure. Fetal complications are mainly due to teratogenic effects of anticoagulation and other drugs. Risk of hypoxia and intrauterine growth restriction is high in foetuses of women with cardiac disease.

Management of pregnancy following cardiac disease include assessment of the patient's symptoms, cardiac function and her functional capacity and also the type of surgery, valve prosthesis, anticoagulation status and anticoagulation drugs. ${ }^{5}$ Continuing advancements in cardiac surgery and management of pregnancies in patients following cardiac surgery is easier now in view of improved functional capacity of the patient and fewer complications faced.

Nevertheless, close monitoring and insight of the anticipated medical problems in this group of patients is mandatory. There is additional circulatory burden on the heart due to haemodynamic changes of pregnancy which is maximum during intrapartum and immediate post partum. ${ }^{8}$ Circulatory overload and the hypercoagulable state of pregnancy are the two difficult conditions which complicate the management. ${ }^{9}$

The functional status of the woman prior to pregnancy is a better predictor of maternal and fetal outcome. Maternal morbidity is directly correlated with functional class. In this study, we found similar outcome. All the patients in our study belonged to NYHA class I and II. Majority of our study population $(39 \%)$ had mitral valve replacement and the next in order was atrial septal defect repair $(37 \%)$. $2.2 \%$ of the patients had repair for tetrology of fallot and $3.3 \%$ had aortic valve replacement.

Anticoagulation is indicated for patients with prosthetic valves due to risk of thrombosis. Change over from oral coumarins to heparin between 6-12 weeks gestation age, continuation of oral coumarins during the rest of pregnancy and then change over to heparin at 36 weeks is advocated. ${ }^{9,10}$ This is done to avoid the teratogenic effects of coumarin and also as a precaution against postpartum 
haemorrhage and to avoid fetal and neonatal complications due to intraventricular haemorrhage. Heparin is a macromolecular agent and it does not cross placental barrier but the disadvantages are difficulty in administration and monitoring. ${ }^{11} 30.4 \%$ of present study population was on anticoagulation. None of our patients had thromboembolism. Anticoagulant therapy can predispose to haemorrhage. $4.3 \%$ of patients had atonic PPH while

$2.2 \%$ had traumatic PPH. Vaginal haematoma is commonly associated with anticoagulation. Hence meticulous repair of episiotomy and vaginal lacerations by closing in several layers and with hemostatic stitches is advocated.

Of the total birth, $77.2 \%$ of the babies had birth weight more than $2.5 \mathrm{~kg}, 18.4 \%$ had birth weight less than 2.5 $\mathrm{kg} .14$ babies were admitted in NICU. Reasons for NICU admission were low APGAR, respiratory distress syndrome and sepsis. Cause of concern for maternal morbidity were pulmonary edema, cardiac arrhythmia and thromboembolism. In our study group, only one woman had supraventricular tachycardia. ${ }^{8}$ ICU care was required for 6 mothers after delivery.

\section{CONCLUSION}

Maternal and neonatal outcome mainly depends on functional cardiac status of women before conception. Physiological changes in cardiovascular system during pregnancy has a major impact on pregnancy. Early detection or any evidence of signs of failure needs urgent hospitalization. These are the good clinical practice points. Role of detailed history and cardiac examination are mandatory during antenatal checkup and early detection and diagnosis of obstetric risk factors play an important role in management. Institutional delivery is recommended. In this study we emphasize on the importance of multidisciplinary team approach involving cardiologist, obstetrician and neonatologist in the management of women with prior cardiac surgery.

Funding: No funding sources Conflict of interest: None declared

Ethical approval: The study was approved by the Institutional Ethics Committee

\section{REFERENCES}

1. Stirling Y, Woolf L, North WR, Seghatchian MJ, Meade TW. Haemostasis in normal pregnancy. Thromb Haemost. 1984 Oct;52(2):176-82.

2. Maiello M, Torella M, Caserta L, Caserta R, Sessa M, Tagliaferri A, Bernacchi $M$ et al. Minerva Ginecol. Hypercoagulability during pregnancy: evidences for a thrombophilic state. 2006 Oct;58(5):417-22.

3. Pritchard JA. Changes in the blood volume during pregnancy and delivery. Anesthesiology. 1965 JulAug;26:393-9.

4. Kanhere AV, Kanhere VM. Pregnancy After Cardiac Surgery. J Obstet Gynaecol India. 2016 Feb;66(1):10-5.

5. Anthony J, Osman, A, Sani MU. Valvular heart disease in pregnancy. Cardiovasc $J$ Afr. 2016;27(2):111-8.

6. Course and Outcome of Pregnancy after the Heart Surgery. [cited 2018 Feb 27]. Available at http://www.wseas.us/elibrary/conferences/2011/Prague/MEDIC/MEDIC07.pdf

7. Bhatla N, Lal S, Behera G, Kriplani A, Mittal S, Agarwal $\mathrm{N}$, et al. Cardiac disease in pregnancy. Int Fed Gynaecol Obstet. 2003Aug;82(2):153-9.

8. Bhutta SZ, Aziz S, Korejo R. Pregnancy following cardiac surgery. JPMA J Pak Med Assoc. 2003 Sep;53(9):407-13.

9. Ayhan A, Yucel A, Bildirici I, Dogan R. Fetomaternal morbidity and mortality after cardiac valve replacement. Acta Obstetricia et Gynecologica Scandinavica. 2001 Aug 1;80(8):713-8.

10. Nishimura RA, Warnes CA. Anticoagulation during pregnancy in women with prosthetic valves: evidence, guidelines and unanswered questions. Heart. 2015 Jan 8:heartjnl-2014.

11. Sillesen M, Hjortdal V, Vejlstrup N, Sørensen K. Pregnancy with prosthetic heart valves-30 years' nationwide experience in Denmark. Eur J CardioThoracic Surg. 2011 Aug;40(2):448-54.

Cite this article as: Rathore S, Shankar R, Vijjeswarapu AP, Abraham A, Yadav B. A five year retrospective study on maternal and perinatal outcome in pregnancy after cardiac surgery. Int J Reprod Contracept Obstet Gynecol 2018;7:1949-52. 